\title{
Improving the sensitivity of a search for coalescing binary black holes with nonprecessing spins in gravitational wave data
}

\author{
Stephen Privitera, ${ }^{1, *}$ Satyanarayan R. P. Mohapatra, ${ }^{2,3, \dagger}$ Parameswaran Ajith, ${ }^{4, \hbar}$ Kipp Cannon,, 8 \\ Nickolas Fotopoulos, ${ }^{1}$ Melissa A. Frei, ${ }^{2}$ Chad Hanna, ${ }^{6,}$ Alan J. Weinstein, ${ }^{1, * *}$ and John T. Whelan ${ }^{2, \dagger \dagger}$ \\ ${ }^{1}$ LIGO Laboratory, California Institute of Technology, 1200 East California Boulevard, \\ Pasadena, California 91125, USA \\ ${ }^{2}$ Center for Computational Relativity and Gravitation and School of Mathematical Sciences, Rochester \\ Institute of Technology, 85 Lomb Memorial Drive, Rochester, New York 14623, USA \\ ${ }^{3}$ Department of Physics, Syracuse University, Syracuse, New York 13244, USA \\ ${ }^{4}$ International Centre for Theoretical Sciences, Tata Institute of Fundamental Research, \\ Bangalore 560012, India \\ ${ }^{5}$ Canadian Institute for Theoretical Astrophysics, 60 St. George Street, Toronto M5S 38H, Canada \\ ${ }^{6}$ Perimeter Institute for Theoretical Physics, 31 Caroline Street North, Waterloo N2L 6B9, Canada
}

(Received 23 October 2013; published 8 January 2014)

We demonstrate for the first time a search pipeline with improved sensitivity to gravitational waves from coalescing binary black holes with spins aligned to the orbital angular momentum by the inclusion of spin effects in the search templates. We study the pipeline recovery of simulated gravitational wave signals from aligned-spin binary black holes added to real detector noise, comparing the pipeline performance with aligned-spin filter templates to the same pipeline with nonspinning filter templates. Our results exploit a three-parameter phenomenological waveform family that models the full inspiral-merger-ringdown coalescence and treats the effect of aligned spins with a single effective spin parameter $\chi$. We construct template banks from these waveforms by a stochastic placement method and use these banks as filters in the recently developed GSTLAL search pipeline. We measure the observable volume of the analysis pipeline for binary black hole signals with $M_{\text {total }}$ and $\chi \in[0,0.85]$. We find an increase in observable volume of up to $45 \%$ for systems with $0.2 \leq \chi \leq 0.85$ with almost no loss of sensitivity to signals with $0 \leq \chi \leq 0.2$. We also show that the use of spinning templates in the search pipeline provides for more accurate recovery of the binary mass parameters as well as an estimate of the effective spin parameter. We demonstrate this analysis on 25.9 days of data obtained from the Hanford and Livingston detectors in LIGO's fifth observation run.

DOI: $10.1103 /$ PhysRevD.89.024003

PACS numbers: 04.30.-w

\section{OVERVIEW}

The coalescence (late-stage inspiral, merger, and ringdown) of binary black hole (BBH) systems is one of the many promising sources of gravitational waves expected to be detectable by ground-based detectors such as LIGO [1] and Virgo [2]. The observation of such systems in gravitational waves will serve as a direct probe of general relativity in the strong-field, highly dynamical regime [3] and have significant implications for the mechanism of their formation through measurements of their coalescence rate as a function of their masses and spins [4].

The components of a coalescing binary black hole system are expected to have significant spins [5-7]. Binary black holes formed through ordinary binary stellar

\footnotetext{
sprivite@ligo.caltech.edu

†satya@astro.rit.edu

*ajith@icts.res.in

\$kcannon@cita.utoronto.ca

`channa@perimeterinstitute.ca

ajw@ligo.caltech.edu

john.whelan@ligo.org
}

evolution from two massive progenitor stars are expected to have spins that are nearly aligned with their orbital angular momentum [8]. On the other hand, black hole binaries formed directly through dynamical capture in dense stellar environments, such as globular clusters, will typically have spins arbitrarily oriented relative to each other and to the orbital angular momentum [9]. Which of these processes contribute to the formation of binary black holes and by how much is highly uncertain [10]. Measuring the rate, masses, and spins of coalescing BBHs therefore will directly inform the processes by which these systems form, the rate at which these processes proceed, and, in the case of common binary evolution, the properties of progenitor systems which eventually become BBHs.

The component spins of a binary black hole system are encoded in the gravitational wave signal emitted during coalescence [11] and are measurable from the observation of these signals [12]. Binary systems with arbitrarily oriented component spins undergo precession of the orbital plane. This precessional behavior manifests in the gravitational wave signal as a quasiperiodic modulation of the signal's amplitude and phase. On the other hand, binaries with 
spins aligned with the orbital angular momentum do not exhibit precession, and no such modulation is present in their gravitational wave signal. Instead, aligned spin systems exhibit nonperiodic (or "secular") contributions to the amplitude and phase of their gravitational wave signals, and these effects make possible, in principle, the discrimination between aligned-spin and nonspinning systems. Since the magnitude and orientation of the spins relative to the orbital angular momentum are determined by the processes that create these binary systems, distinguishing between these three cases (precessing, nonprecessing, and nonspinning) in gravitational wave observations allows for the interpretation of these observations in terms of the astrophysical mechanism which creates these systems.

With the availability of predictions for the waveforms emitted during coalescence, searches for gravitational waves from BBHs profit greatly from the use of matched filtering to sift through the data. The matched filter gives the maximal signal-to-noise ratio (SNR) among all possible linear filters in the ideal case of a Gaussian-distributed background, assuming that the template corresponds exactly to the signal potentially in the data. In practice, we do not know a priori the exact parameters of the binary system which creates the signal, and we therefore analyze the data using a large number of waveforms with discretely sampled parameters. The true signal typically will not be identically one of the filter templates, either because of the discreteness of the bank or because of imperfect waveform modeling, in which case the matched filtering technique becomes suboptimal. However, if the true signal is similar enough to one of the filter templates, the expected loss of SNR due to these effects may be negligibly small.

The data analysis problem is significantly complicated by non-Gaussian artifacts present in gravitational wave data from real detectors. In this case, the matched filter SNR is not optimal for detection, and we require additional background-signal discrimination techniques. For example, recent LIGO and Virgo searches [13-15] have benefited greatly from the use of a $\chi^{2}$ statistic [16], which uses the triggered template to subtract out the putative signal from the data and test whether the resulting data stream is consistent with Gaussian noise. As with the matched filter, the efficacy of this statistical test relies crucially on having an accurate waveform model for the signal. The $\chi^{2}$ test can easily mistake a large mismatch between the template and the signal as being due to non-Gaussian noise, and the event could consequently be missed by the search.

Given that BBH systems probably have significant spins and that the search sensitivity depends strongly on having accurate waveform models, the inclusion of spin effects in search templates has for good reason been a long-standing goal in the field [17-26]. Yet none of the previous attempts to include spin effects in templates have resulted in improved search sensitivity. In Ref. [17], an analysis of data from LIGO's third science run demonstrated for the first time the use of spinning templates [31] in an actual search, using a phenomenological model for the inspiral phase of precessing compact binaries. However, it was later shown [18] that the use of spinning templates in this search increased the false alarm rate so much as to offset the gains in signal to noise obtained by the use of these templates. Thus, for a given level of detection confidence, the pipeline used for this search with spinning templates was unable to recover more spinning systems than the same analysis using nonspinning templates. The results of these studies motivated the decision to neglect spin effects in search templates in the most recent LIGO and Virgo searches for BBH coalescence [13-15,27], and to date no other search of LIGO and Virgo data has used spinning templates.

The null result in Ref. [17] was attributed to the elevation in the rate of background events owing to the larger number of templates required to cover the spinning waveform parameter space. It was immediately realized that to make the use of spinning templates in an analysis beneficial, better signal consistency tests would have to be developed and implemented to suppress the increased background event rates. In particular, the highly effective $\chi^{2}$ test used in the contemporary LIGO search with nonspinning templates [27] was never integrated into the spinning search pipeline and probably at a severe cost for the results of the sensitivity analysis. Following this work, two other pipelines were developed $[19,20]$ which included spinning templates based on a modified phenomenological model for singlespin binaries [22], but neither analysis demonstrated conclusively that the techniques would improve the search sensitivity. Neither of these investigations examined the use of signal consistency tests for suppressing the background.

A couple of pipeline implementation issues arose from these investigations, which ultimately concern the question of having a measure for the "distance" between nearby templates. The first was the problem of defining coincidence between triggers in different detectors. In the twodimensional mass parameter space, elegant and rigorous techniques exist for defining when the parameters of two triggers are close enough to be considered the same [28]. However, it was not clear at the time how to extend this method to define a robust coincidence criterion in higher-dimensional parameter spaces. The analyses in Refs. [17,20] defined coincidence between triggers in terms of the standard mass coincidence criterion proposed in Ref. [28] together with a simple interval cut on the remaining parameters. The study in Ref. [19] was for a coherent analysis for which the question of coincidence is irrrelevant.

Another problem that comes with having a larger template parameter space is that of efficiently placing templates to minimize the loss of SNR arising from the discreteness of the template bank. Recent LIGO and Virgo compact binary searches, which used nonspinning templates, have relied on a lattice placement technique known to select the fewest number of templates for a given tolerance 
of SNR loss. [29-32]. This technique relies on having knowledge of certain special parameters for which the mismatch between two neighboring templates is the ordinary Euclidean norm applied to these parameters. It is then straightforward to construct a regular lattice in these coordinates to guarantee a specified maximal loss of SNR for any signal which lies between the templates. However, the method requires one to determine which coordinates, if any, are appropriate for the lattice construction. In largerdimensional parameter spaces or when using waveforms which include effects beyond the inspiral portion of the coalescence, we often do not have any known special parameters in which to form the lattice, and other approaches to template placement become necessary.

In the studies discussed above, two main approaches were taken to construct banks of spinning templates. The first is a simple stacking method in which one lays out a grid of points in the spin parameters and for each point in the grid applies to the lattice technique to lay out templates in $m_{1}$ and $m_{2}$. The main limitation of this technique is that it is not known a priori how fine the grid spacing needs to be to fully cover the space. One determines the required spacing through simple trial and error. Additionally, this approach provides very little assurance that the resulting template bank is close to the minimal size bank needed to cover the space.

In Ref. [17], the authors also explored the use of a stochastically generated bank and found it to give significantly fewer templates than the stacking approach. More recently, several groups have conducted thorough and systematic studies of the stochastic template placing techniques [33,34] and the outlook is quite promising [26]. Stochastic placement techniques are applicable to a wide variety of waveform approximants, requiring no prior knowledge of special parameters and extending straightforwardly to higher dimensions. We discuss these template placement methods in more detail in the following section.

Here we revisit the problem of using spinning waveform models as search templates. Our analysis uses the threeparameter IMRPhenomB waveform family [35] modeling the inspiral, merger, and ringdown of binary black hole systems with aligned spins. This waveform family captures the dominant effects of aligned spin with a single effective spin parameter $\chi$ defined below. We construct template banks from these waveforms using a generic, extensible stochastic placement infrastructure SBANK [26] implemented in the LAL gravitational wave data analysis library [36] and incorporate the SBANK infrastructure into the recently developed GSTLAL pipeline [37]. This pipeline uses identical template banks in each detector and imposes an exact mass and spin parameter coincidence criterion between detectors. Additionally, the analysis employs a computationally efficient autocorrelation $\chi^{2}$ statistic to suppress non-Gaussian background fluctuations. We apply the search pipeline to 25.9 days of detector noise obtained from the initial LIGO detectors at Hanford and Livingston. We demonstrate for this data set an increase in the sensitive volume for signals with $\chi \in[0.2,0.85]$ of up to $45 \%$ with only a moderate loss of sensitivity to signals with $\chi \in$ $[0,0.2]$ when the filter templates include the effects of aligned spin.

In a few years, the advanced LIGO and Virgo detectors will come online with much better sensitivity compared to initial LIGO detectors $[38,39]$. As these detectors will have significantly improved sensitivity and bandwidth, the importance of including spin in waveform templates will only become greater. While advanced LIGO and Virgo may bring with them non-Gaussian backgrounds unlike those seen in initial LIGO, we emphasize that our results here for the improved sensitivity to spinning signals are demonstrated in real detector data from initial LIGO and in doing so directly confront the hazards of realistic non-Gaussian detector behavior. The template placement infrastructure and analysis pipeline we use in this work are readily scalable to the data analysis requirements of advanced LIGO and Virgo sensitivities, and we anticipate that the improvements shown here will be readily transferable to BBH searches in advanced LIGO and Virgo data.

In the following section, we briefly review commonly used template placement strategies and motivate our adoption here of the stochastic approach. We then apply the stochastic placement method to construct aligned-spin and nonspinning template banks. In doing so, we also identify the regions of parameter space with the greatest potential for improvement in sensitivity by the inclusion of spin. Focusing in on these regions, we then demonstrate the implementation of our template banks in the GSTLAL search pipeline. We find for a fixed false alarm rate that the pipeline analysis with aligned-spin templates has an observable volume that is $95 \%$ to $145 \%$ that of the observable volume for same pipeline with nonspinning templates in the considered parameter space. Furthermore, we show that the use of aligned-spin templates allows for more accurate mass and spin parameter recovery in the pipeline. We conclude this paper with a discussion of other compact binary search problems to which we hope the methods detailed here will apply.

\section{STOCHASTIC TEMPLATE PLACEMENT}

As mentioned in the introduction, a matched filter search for binary black hole coalescences requires the use of a bank of template waveforms with discretely sampled source parameters. In this section, we review lattice and stochastic template placement techniques and define the notions we will use in the subsequent section to quantitatively describe the efficacy of a given template bank for the detection of a population of target signals

A useful quantitative measure for the effectiveness of a template bank toward capturing the SNR for a target signal 
population is the fitting factor [40], which we now define. Given two waveforms $h_{1}(t)$ and $h_{2}(t)$, we define the overlap between two waveforms $h_{1}(t)$ and $h_{2}(t)$ by the integral

$$
\left\langle{ }_{1} \mid h_{2}\right\rangle=2 \int_{f_{\text {low }}}^{\infty} \frac{\tilde{h}_{1}(f) \tilde{h}_{2}^{*}(f)+\tilde{h}_{1}^{*}(f) \tilde{h}_{2}(f)}{S_{n}(f)} d f,
$$

where $S_{n}(f)$ is the (one-sided) power spectral density of the detector noise, $f_{\text {low }}$ is the low-frequency cutoff, the tilde denotes the Fourier transform of the waveform, and the asterisk denotes complex conjugation [41]. We denote normalized waveforms with a hat so that $\left\langle\tilde{h}_{i} \mid \tilde{h}_{i}\right\rangle=1$. If we denote the template bank by $B=\left\{\hat{h}_{i}\right\}_{i=1}^{N}$ and $\hat{h}_{\vec{\lambda}}$ is some target signal with arbitrary source parameters $\vec{\lambda}$, then we define fitting factor of the bank toward this signal by

$$
\operatorname{FF}(\vec{\lambda} ; B)=\max _{i, t}\left\langle\hat{h}_{i} \mid \hat{h}_{\vec{\lambda}}\right\rangle,
$$

where the maximization is taken not only over the templates in the bank but also over the time translation of the templates. In the construction of a template bank, the goal generally is to achieve the highest possible fitting factors with the fewest possible templates.

Currently implemented LIGO-Virgo matched filtering searches for BBHs are based on a lattice approach for placing the templates $[42,43]$, and similar techniques are being developed for advanced generation searches [23-25]. This technique relies on the existence of coordinates $\vec{\lambda}^{\prime}=f(\vec{\lambda})$ such that at any point $\vec{\lambda}$ in the source parameter space the fitting factor between the bank $B$ and the signal $h_{\vec{\lambda}}$ is approximately

$$
\operatorname{FF}(\vec{\lambda} ; B) \approx 1-\min _{i}\left|f\left(\vec{\lambda}_{i}\right)-f(\vec{\lambda})\right|^{2}
$$

for $\left|\vec{\lambda}_{i}-\vec{\lambda}\right| \ll 1$. One can then place the templates on a regular lattice in these coordinates to guarantee a minimal loss of SNR [29,31]. The lattice technique is highly computationally efficient but requires one to determine the appropriate coordinates in which to lay down the templates.

In the inspiral-merger-ringdown regime, however, no such coordinates are known, and the lattice techniques cannot be reliably applied to place templates. Therefore, in this study we adopt a stochastic approach [33,34] to place templates in the mass and spin parameter space of binary black hole systems. In this approach, template parameters are proposed randomly, and the proposed waveform's fitting factor is computed against some initial seed bank. A threshold value for the minimal fitting factor is chosen (here we take $\mathrm{FF}_{\min }=0.97$ ), and if the proposed template does not achieve a fitting factor larger than this value, then the proposal is added to the bank. This extended bank becomes the seed for the next iteration. Otherwise, the proposal is discarded, and the same bank is used for the seed of the next iteration. The iteration continues until the rejection rate of proposals becomes sufficiently high.

We reuse a recently developed generic and extensible implementation of this algorithm, referred to as SBANK, which has previously proven effective in constructing template banks for lower mass compact binary systems with spin [26]. The stochastic placement technique is robust, and we show here that it works well in three dimensions. While the implementation of this technique to higher dimensions is straightforward, the required convergence time for generating a higher-dimensional bank may become impractical, and the resulting template banks can be significantly larger than the theoretically optimal template bank size [34]. Furthermore, even in a small number of dimensions, long waveforms can lead to computationally costly Fourier transforms and considerably slow down the convergence time. In such cases, it is possible to side step the numerical fitting factor calculation with an analytic approximation to the fitting factor as in Eq. (3) and demonstrated in Ref. [26].

\section{TEMPLATE BANK EFFECTUALNESS}

Here we determine the regions in parameter space which have the greatest potential for improvement in SNR recovery by the inclusion of aligned-spin waveforms in a search template bank. Our study employs the IMRPhenomB waveform family [35], which models the late-inspiral [that is, $f_{\mathrm{GW}} \gtrsim 10^{-3} /\left(G M_{\mathrm{total}} / c^{3}\right)$, where $f_{\mathrm{GW}}$ is the dominant mode gravitational wave frequency], merger, and ringdown stages of the coalescence of binary black holes with aligned spins. The waveform family is parametrized by the component masses $m_{1}$ and $m_{2}$ of the binary and a single effective spin parameter $\chi$. The effective spin parameter captures the dominant waveforms effects arising from aligned spin and reduces the dimensionality of the intrinsic parameter space from four to three. The effective spin is defined by

$$
\chi \equiv \frac{m_{1} \chi_{1}+m_{2} \chi_{2}}{m_{1}+m_{2}},
$$

where $\chi_{1}$ and $\chi_{2}$ are the dimensionless spins of the component black holes.

The IMRPhenomB waveform model consists of a parametrized phenomenological fit to hybrid waveforms constructed from numerical relativity simulations of the late inspiral, merger, and ringdown of binary black holes matched to a post-Newtonian approximation describing the early inspiral. As such, the validity of these waveforms has restrictions on the mass ratio and spins based on the availability of numerical simulations with which to fit. Specifically, the IMRPhenomB family is expected to be accurate only for low to moderate mass ratios and spins. Hence, in this study, we consider only binaries for which $1 \leq m_{1} / m_{2} \leq 4$ and $-0.5 \leq \chi<0.85$. 
We choose to further focus only on the regions in the parameter space where the merger and ringdown stages are important for detection. For an initial LIGO design sensitivity, the effects of merger and ringdown begin to contribute significantly to the SNR when the total mass of the binary exceeds $M_{\text {total }} \approx 12 M_{\odot}$ [44]. For lower mass systems, accurate and generically spinning post-Newtonian waveforms are available [36] and can be used to give a more detailed understanding of the effects of spin on the search. We therefore consider only systems with $M_{\text {total }} \geq 10 M_{\odot}$, giving a small safety factor between the transitional region and considering the degeneracy between the mass and spin parameters.

Since the finite size of neutron stars can have a significant impact on the gravitational waveform observed in the merger phase of coalescence, we restrict our attention to binary black holes and take $m_{i}=3 M_{\odot}$ as the minimal component mass. We note that from astrophysical considerations, neutron stars in coalescing compact binaries are not expected to have large spins. Further, from physical considerations of the possible neutron star equations of state, the dimensionless spin for a neutron cannot exceed $\sim 0.7$ without undergoing tidal disruption.

We used the SBANK implementation of the stochastic placement method described in Sec. II to generate a bank of IMRPhenomB templates with $\chi=0$ using the above mass parameter restrictions and $f_{\text {low }}=40 \times \mathrm{Hz}$. We then computed the fitting factors of this template bank toward aligned spin signals in the same $m_{1}-m_{2}$ parameter space assuming the initial LIGO design sensitivity shown in Fig. 2. In Fig. 1(a), we show that a template bank with $\chi=0$ already captures greater than $97 \%$ of the possible SNR over a wide mass and spin range. In particular, we note that the $\chi=0$ bank covers signals with $\chi<0$ down to roughly $\chi \sim-0.25$ over the entire mass range. From astrophysical considerations of binary evolution, spins positively aligned with the orbital angular momentum are considered the more likely scenario for binary black holes [8]. Given these factors, along with the potential for artifacts in the waveforms at large negative $\chi$, we develop our search using only $\chi \geq 0$ templates. Note that since $\chi$ is a mass-weighted sum of the two component spins, this restriction does not necessarily exclude the possibility that one of the black holes has an antialigned spin. We also see that as the total mass of the target system increases, the fractional loss of SNR incurred from neglecting spin decreases. This effect is due to the fact that higher mass systems merge at lower frequencies and have fewer cycles in the LIGO sensitive band, and consequently the matched filtering is more tolerant of imperfect templates. The apparent loss of SNR at high mass and $\chi<0$ is an artifact of the boundary of the template bank. Negative alignedspin systems have shorter waveforms, which, in the absence of spinning templates, match best with higher mass nonspinning templates. We thus expect that for systems with

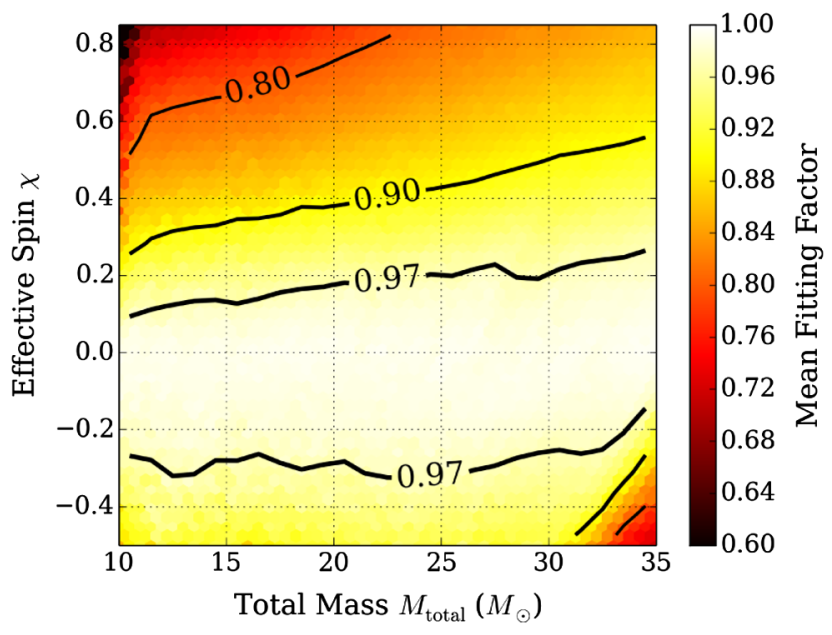

(a) $\chi=0$ Template Bank

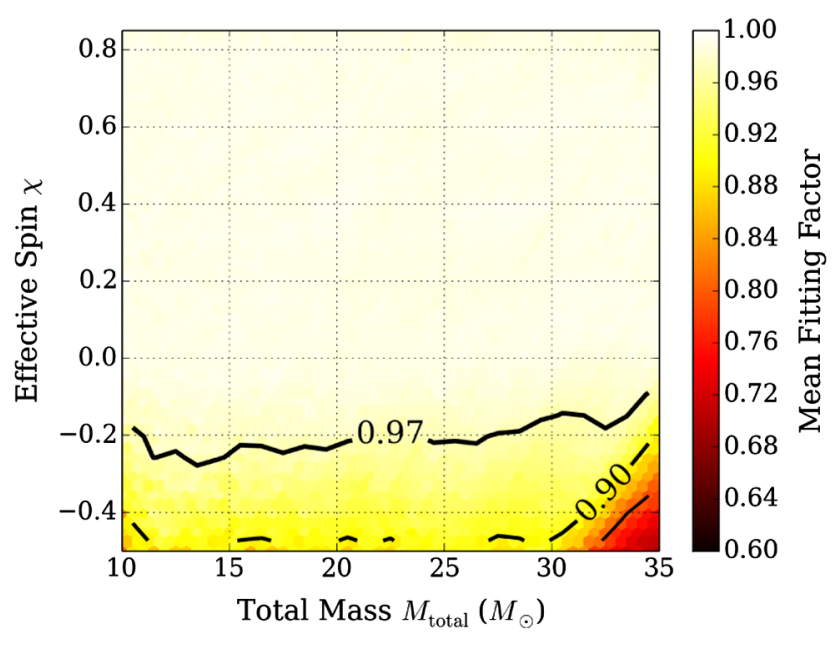

(b) $\chi \geq 0$ Template Bank

FIG. 1 (color online). Capturing aligned-spin effects in template banks. Above we show the expected fractional signal-to-noise recovery for a population of aligned-spin binary black holes using a bank of IMRPhenomB waveforms with (a) $\chi=0$ and (b) $\chi \geq 0$. The solid lines indicate the approximate fitting factor contours in the $M_{\text {total }}-\chi$ plane, averaging over the mass ratio dimension with $1 \leq m_{1} / m_{2} \leq 4$. The template banks are both constructed with the stochastic placement method described in Sec. II assuming the design iLIGO sensitivity [45] with $f_{\text {low }}=40 \mathrm{~Hz}$. We find that with this sensitivity, a template bank that neglects spin achieves fitting factors exceeding the nominal $\mathrm{FF}_{\min }=0.97$ from aligned-spin systems over a wide region of parameter space, spanning roughly $-0.25 \leq \chi \leq 0.2$ over the entire mass range. As the mass of the system increases, the loss of signal to noise incurred from neglecting spin becomes small, and we therefore do not consider systems with total masses exceeding $M_{\text {total }}=35 M_{\odot}$. The $\chi=0$ bank has $\sim 700$ templates, whereas the $\chi \geq 0$ bank has $\sim 3000$ templates.

total masses exceeding $M_{\text {total }}=35 M_{\odot}$, the benefits of including spin effects will be small.

In Fig. 1(b), we demonstrate the coverage of the parameter space obtained by including only waveforms 


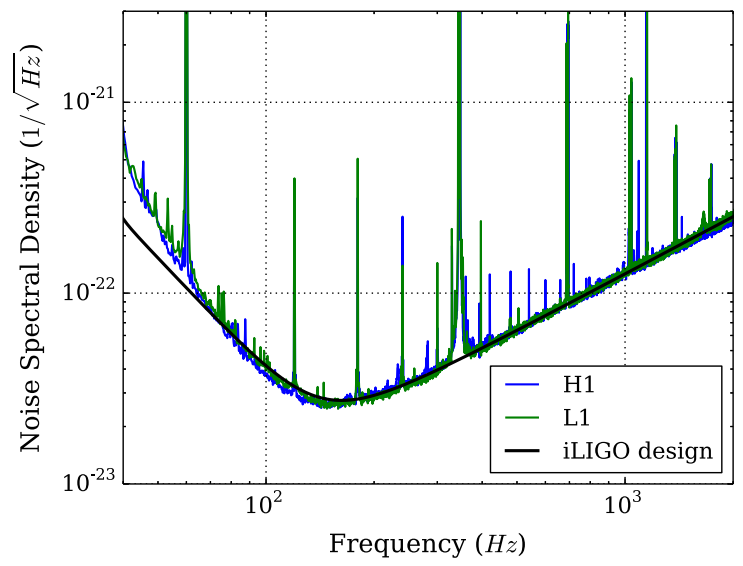

FIG. 2 (color online). Detector sensitivities. Here we show characteristic noise spectral density curves for data from the S5 observational run of the two detectors H1 and L1 used to supply noise to which simulated signals are added in this study [46]. The black line shows the initial LIGO design sensitivity, which we used to compute template bank effectualness in Fig. 1.

for non-negative aligned spins $(\chi \geq 0)$ in the template bank. The improvement in SNR recovery obtained by using such a bank comes at the cost of having more than three times as many templates in the bank. For the nonspinning case, we constructed a bank with $\sim 700$ templates, while to cover the positively aligned signals, we require $\sim 3000$ templates. The increase in the number of templates will increase the number of background triggers, and detecting a signal at a given false probability requires raising the SNR threshold used for detection.

The expected SNR scales inversely with distance to the source, and the detection rate scales as the cube of the sensitive distance. Therefore, the fractional increase in detection rate due to the inclusion of spinning waveforms in the template bank should be given by

$$
\frac{V_{\text {spin }}}{V_{\text {nonspin }}}=\left(\frac{\mathrm{FF}_{\text {spin }}}{\mathrm{FF}_{\text {nonspin }}}\right)^{3} \text {, }
$$

where $\mathrm{FF}_{\text {nonspin }}$ and $\mathrm{FF}_{\text {spin }}$ are the fitting factors for the nonspinning and spinning template banks, respectively, to the target signal. These statements regarding the increase in detection rate assume that the only gain in sensitivity comes from the SNR. In practice, our data also contain non-Gaussian artifacts, and the SNR is not an optimal detection statistic, as mentioned above.

The characteristics of the background can change in complicated ways when new template waveforms are introduced to a search. The results presented in Fig. 1 do not reflect the impact of non-Gaussianity in the data, nor to they capture the effects of multidetector coincidence requirements, the use of $\chi^{2}$ statistics, increased false alarms due to larger template banks, or other effects which are important in realistic search pipelines. In the following section, we describe the implementation of these spinning template banks in a search pipeline. We show that even in non-Gaussian data, we are able to sufficiently suppress the extra background to achieve a net gain in the search sensitivity.

\section{IMPLEMENTATION OF SPIN EFFECTS IN A SEARCH OF GRAVITATIONAL WAVE DATA}

We now demonstrate the use of the template banks constructed in Sec. III as filters in the GSTLAL search pipeline [37]. Using these template banks, we measured the sensitivity of the pipeline to a simulated population of more than 200,000 binary black holes. Here we compare the mean sensitive distance of the pipeline analysis when using a bank of aligned-spin templates against that of an otherwise identical analysis which uses nonspinning templates. Our simulated binary black hole systems were populated with a uniform distribution in mass ratio, total mass, and effective spin with $m_{1} / m_{2} \in[1,4], M_{\text {total }} \in[15,25] M_{\odot}$, and $\chi \in[0,0.85]$. The simulated waveforms that were added to the detector noise were computed using the same IMRPhenomB approximation used for the template waveforms. We conducted our study on 25.9 days of coincident detector noise obtained from observations of the Hanford and Livingston detectors during LIGO's fifth science run. Typical strain sensitivities for these two detectors during this science run are shown in Fig. 2.

In Fig. 3, we show the measured sensitivities of our two analyses in terms of the mean distance accessible to each search as a function of the false alarm rate threshold. We show our results only for systems with total masses in the range $M_{\text {total }} \in[15,25] M_{\odot}$ to avoid complications associated with the boundaries of the template banks, which covered the range $M_{\text {total }} \in[10,35] M_{\odot}$. As expected and demonstrated in Fig. 3(c), we find that the greatest improvement in sensitivity is for target systems with high effective spins. As seen in Fig. 3(d), the volume improvement, and therefore the increase in detection rate, can be as high as $45 \%$ for these highly spinning systems. We emphasize the nontrivial result shown in Fig. 3(a) that for weakly spinning target systems $(\chi \leq 0.2)$ the analysis with spinning templates and the analysis with nonspinning templates have comparable sensitivities, with the aligned-spin template analysis achieving at worst 95\% of the sensitive volume of the nonspin template analysis. The apparent loss of detection rate in the small effective spin regime is only applicable if we are wrong in our expectations that black holes have significant spins. Otherwise, we expect this search method to increase the overall detection rate of spinning BBH systems, provided that these spins are aligned. These results demonstrate for the first time an analysis of real detector data, which is made more sensitive to spinning signals by the use of spinning templates compared to the same analysis performed with nonspinning templates. 


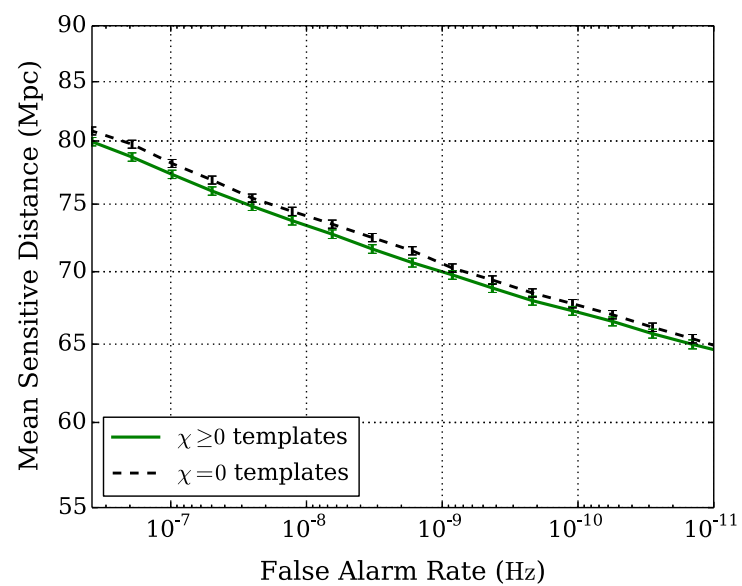

(a) Injections with $0 \leq \chi \leq 0.2$

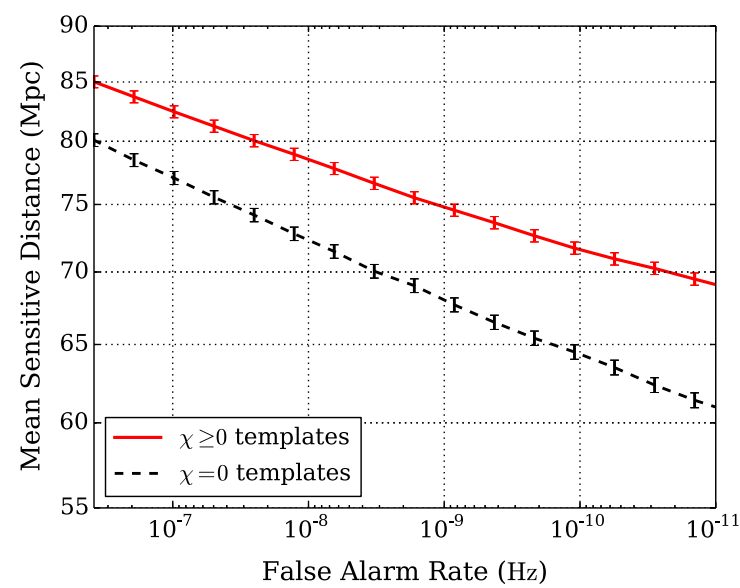

(c) Injections with $0.5 \leq \chi \leq 0.85$

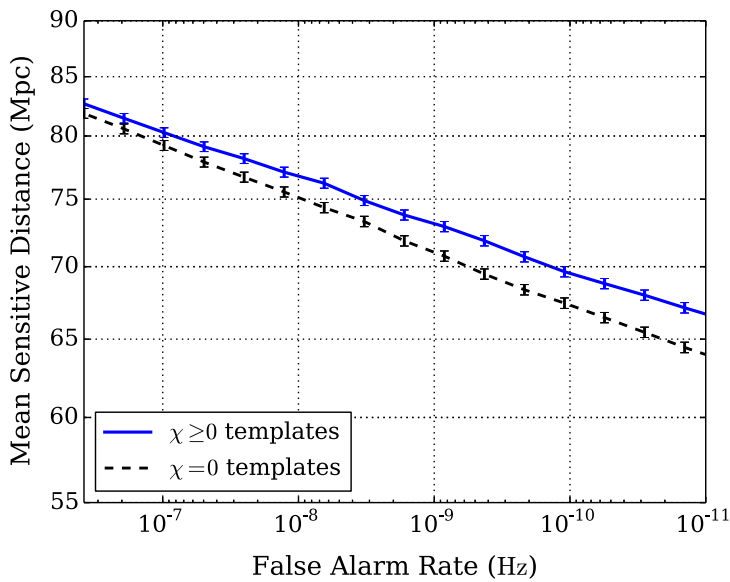

(b) Injections with $0.2 \leq \chi \leq 0.5$

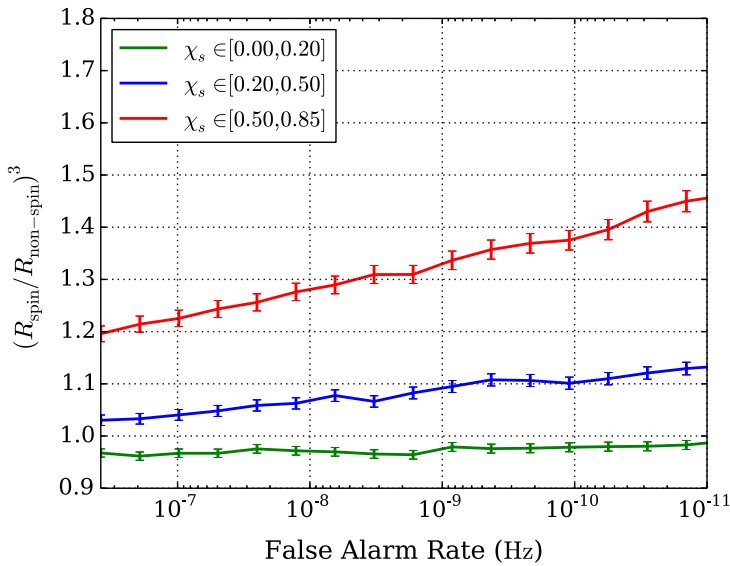

(d) Volume Ratios

FIG. 3 (color online). Comparison of search sensitivities as a function of false alarm rate threshold. Here we compare the sensitivities to aligned spin systems with $M_{\text {total }} \in[15,25] M_{\odot}$ for an analysis which used templates with $\chi \geq 0$ and an analysis which used templates with $\chi=0$. The template banks each covered the mass range $M_{\text {total }} \in[10,35] M_{\odot}$. In (a-c), we show the absolute sensitivities for these analyses in terms of the average distance to which the analyses identify an injection with a trigger above a given false alarm rate threshold. In (d), we show the ratios of the sensitive volumes for each of the three spin bins. We find that for injections with $\chi \geq 0.2$, the spinning search observes a larger sensitive volume than the nonspinning search for all false alarm rates by as much as $45 \%$. For injections with $0 \leq \chi \leq 0.2$, we observe a small but statistically significant decrease in sensitive volume on the order of $5 \%$ incurred by the use of spinning templates.

The analysis performed here differs from previous attempts toward the inclusion of spin effects in search templates in a number of ways. First, this analysis makes use of a template family that captures the effect of nonprecessing spins by using a small number of physical parameters, which allows us to construct a simple three-dimensional template bank. Recent studies have suggested that such template banks are effectual for a significant fraction or precessing binaries as well $[26,35,47]$. This is in sharp contrast with the earlier work, which either used phenomenological parameters to capture spin effects $[17,21]$ or methods to maximize the SNR over a number of extrinsic parameters that produced elevated background [22].
This analysis also used an autocorrelation $\chi^{2}$ statistic, analogous to the time-frequency statistic $\chi^{2}$ developed in Ref. [16] used in recent LIGO and Virgo compact binary searches [13-15]. The autocorrelation statistic is based on the principle that the SNR time series obtained from filtering data which contains a signal against a template that closely matches the signal is approximately equal to the autocorrelation function of the template plus noise. Subtracting the template autocorrelation from the SNR time series and computing the residual noise power gives a measure of the consistency of that data with the signal model.

As discussed in the introduction, previous studies on the inclusion of spin effects in template waveforms suffered in part due to the lack of sufficiently strong signal consistency 
tests to reject triggers occurring due to non-Gaussian artifacts in the data. We suggest that the autocorrelation test used here was instrumental toward achieving our results and encourage the development and implementation of other signal-based consistency tests, which could be added to this analysis to improve upon these results (one such consistency test, known as the bank veto [48], is currently being tested within the GSTLAL pipeline). We also point out that the autocorrelation consistency test is appealing from a computational point of view since once a trigger has been produced by the pipeline the needed SNR time series is already available in memory and the calculation comes at nearly no extra cost.

We have also taken a simplistic, but seemingly quite powerful, approach to the matter of defining the coincidence of triggers between detectors. For coincidence in time, we follow the standard interval approach, requiring that triggers occur within $3 \mathrm{~ms}$ of each other after correcting for maximum light travel time between the detectors. For mass and spin coincidence, however, we require that triggers in each detector have identical parameters. This choice is possible in the GSTLAL framework since the same template bank is used for all detectors and all times in the analysis. Previous LIGO and Virgo searches for compact binary coalescence have used template banks for which the parameters depend on the local power spectral density of a detector, resulting in template banks which are different in each detector and at different times. In the latter implementation, the coincidence criterion must allow for some small mismatch in the trigger parameters from different detectors. Recent searches using two-parameter nonspinning template banks have achieved this tolerance using estimates of the expected uncertainty in parameter recovery to define a small error region [28], but the generalization of this technique to higher-dimensional parameter spaces is not straightforward, and the exact size of the error region typically requires careful tuning in order to be effective. On the other hand, the exact parameter coincidence feature of the GSTLAL search pipeline generalizes trivially to higher-dimensional parameter spaces and requires no tuning. The results here suggest the exact coincidence criterion is a strong discriminator between background and signal, but we do not systematically examine the relative merits of these two approaches.

Ultimately, the key to improving the sensitivity of a search pipeline by the inclusion of more physical effects in the search templates is the ability to manage the background trigger rates while exploiting the elevation of the signal. The methods described here have proven successful in mitigating the background elevation relative to the signal to obtain a net gain in sensitivity. We have highlighted in this section just two features of the GSTLAL pipeline which are manifestly different from other studies and lie at the core of the background rejection techniques currently implemented in the pipeline. Given that the GSTLAL pipeline has not previously been used for an analysis of this type, there are of course many other differences between this work and previous studies, but isolating the particular features which made these results possible is a difficult task.

\section{PARAMETER RECOVERY}

There are robust, dedicated parameter estimation algorithms which can extract the parameters of a signal in gravitational wave data with high accuracy [49], after the signal is found in a detection algorithm. These algorithms are computationally intensive, requiring a detailed exploration of the full, high-dimensional binary parameter space based on Bayesian analysis with Markov-chain Monte Carlo or nested sampling methods. Parameters of a signal can be inferred rapidly, albeit with less accuracy, from a matched filter detection algorithm from the identified template parameter. In this paper, although we focus on the detection performance, we also report two parameter extraction properties of the search algorithm so as to understand any possible biases in the search algorithm.

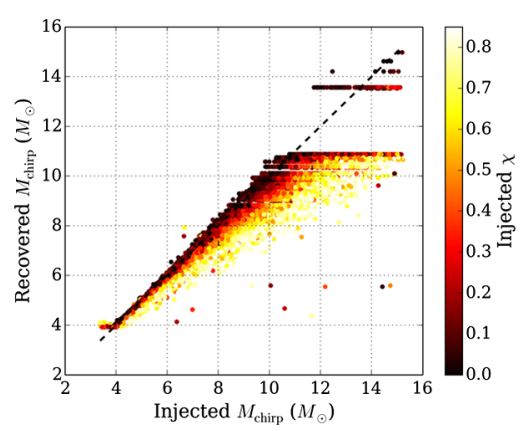

(a) $\chi=0$ Template Bank

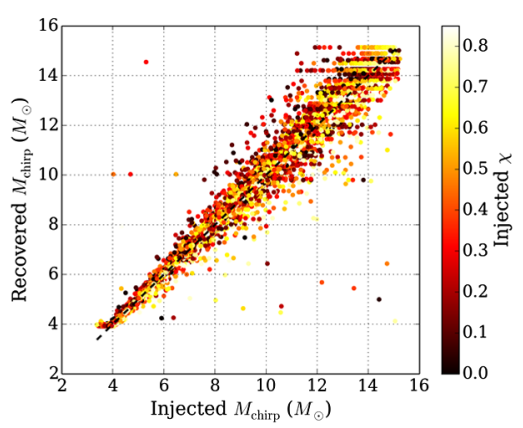

(b) $\chi \geq 0$ Template Bank

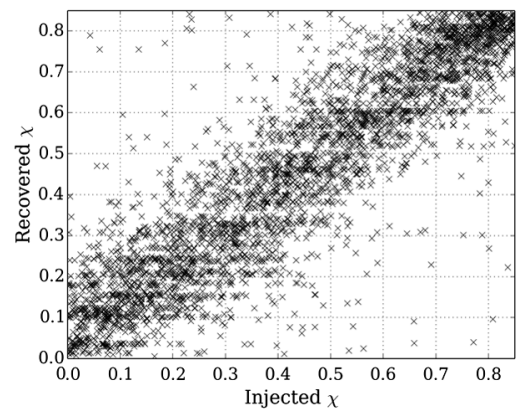

(c) $\chi \geq 0$ Template Bank

FIG. 4 (color online). Improvement in parameter recovery. In addition to improving the sensitive search volume, the inclusion of spin effects helps to curtail systematic biases in the recovery of template parameters. Here we demonstrate the improvement in chirp mass recovery when spin is included in the templates. The left panel corresponds to the analysis with nonspinning templates while the middle and right panels correspond to the analysis with aligned-spin templates. 
In Fig. 4(a), we show the bias introduced in the recovery of the chirp mass parameter when signals from positively aligned systems are searched for using nonspinning templates. The bias indicates a degeneracy in the parameter space. Positively aligned spins allow the binary to evolve to much higher frequencies (orbital hang up [50]), and this increases the length of the waveform, as compared to its nonspinning counterpart. Thus, high mass positively spinning systems may match best with lower mass nonspinning systems. However, as shown in Fig. 4(b), when the proper spin effects are included in the templates, the parameter bias is substantially decreased. Furthermore, as seen in Fig. 4(c), we find that we can recover the injected effective spin parameter with moderate accuracy, when we include the spin in the search templates.

\section{CONCLUSION}

We have demonstrated the first implementation of a detection pipeline for coalescing binary black holes, which uses spinning templates to achieve in real detector noise a greater sensitivity to spinning signals. This analysis used the IMRPhenomB waveform model, which includes the full inspiral, merger, and ringdown phases of the coalescence and additionally models aligned-spin effects with a single effective spin parameter $\chi$. Using this model, we constructed two template banks of binary black holes with $M_{\text {total }} \in[10,35] M_{\odot}$ and $1 \leq m_{1} / m_{2} \leq 4$. In one template bank, we restricted the templates to effective spins with $\chi=0$, and in the other, we included templates with $\chi \geq 0$. These banks were constructed using the stochastic placement infrastructure SBANK and implemented for filtering in the GSTLAL search pipeline. We measured and compared the sensitivity of this pipeline with each of the constructed template banks to IMRPhenomB aligned-spin target signals with $M_{\text {total }} \in[15,25] M_{\odot}$ and $\chi \geq 0$. This sensitivity analysis was conducted with simulated signals added to 25.9 days of real detector noise obtained from the fifth observation run of the Hanford and Livingston detectors.

Our analysis showed an increase sensitive volume of up to $45 \%$ for target systems with $0.2 \leq \chi \leq 0.85$ when aligned-spin effects were included in the templates. We have also reported that with the use of aligned-spin templates in the pipeline, there is the added advantage that the effective spin parameter of the binary black holes can be inferred, and more accurate mass parameter estimation can be achieved. This study is the first demonstration of a full detection pipeline in which the use of spinning templates has been shown increase the sensitivity to aligned-spin signals relative to the same pipeline using nonspinning templates. We emphasize that this improvement is demonstrated in real LIGO detector noise and therefore includes the effects of dealing with non-Gaussian noise artifacts.

The demonstration given here is encouraging and represents an important step toward integrating spin effects in LIGO and Virgo searches for compact binary coalescences. In this work, we restricted our sensitivity measurements to binary black holes with non-negative effective spins and total masses in $[15,25] M_{\odot}$, a very small, though astrophysically favored, portion of the much larger parameter space accessible by LIGO and Virgo observations. Future work will address some of these other regions of the mass and spin parameter space. In particular, we plan to study the importance of spin for searches of lower mass compact binary systems $\left(M<12 M_{\odot}\right)$. Such systems may be well modeled by inspiral-only waveforms, and in this regime there exist accurate waveform models for precessing binaries, which may be particularly important for binary black holes. Ultimately, our primary goal is to establish the viability of the methods presented here for the use of spinning templates in searches of data having noise characteristics similar to the anticipated sensitivities of the advanced LIGO and Virgo detectors. We expect that when the sensitivities of the detectors are such that the lower frequency can be profitably lowered from $40 \mathrm{~Hz}$, as anticipated for the advanced LIGO and Virgo detector era, the gains in sensitivity to spinning signals achievable through the methods presented here will be substantially greater.

\section{ACKNOWLEDGMENTS}

The authors gratefully acknowledge the support of the United States National Science Foundation for the construction and operation of the LIGO Laboratory. This work was supported by NSF Grants No. PHY-0855494 and No. PHY-1207010. P. A.'s research was partially supported by a FastTrack fellowship and a Ramanujan Fellowship from the Department of Science and Technology, India, and by the EADS Foundation through a chair position on "Mathematics of Complex Systems" at ICTS-TIFR. The authors would like to thank those individuals in the gstlal group who wrote the programs used to perform the spinning analyses and Thomas Dent for useful comments on the manuscript. S. R. P. M. would like to thank Prayush Kumar for useful discussion. S. P. would like to thank Deborah Hamm for useful discussion. 
[1] B. Abbott et al. (LIGO Scientific Collaboration), Rep. Prog. Phys. 72, 076901 (2009).

[2] T. Accadia et al. (Virgo Collaboration), JINST 7, P03012 (2012).

[3] N. Yunes and X. Siemens, arXiv:1304.3473.

[4] B. S. Sathyaprakash and B. F. Schutz, Living Rev. Relativity 12, 2 (2009).

[5] C. F. Gammie, S. L. Shapiro, and J. C. McKinney, Astrophys. J. 602, 312 (2004).

[6] R. C. Reis, A. C. Fabian, R. R. Ross, and J. M. Miller, Mon. Not. R. Astron. Soc. 395, 1257 (2009).

[7] J. E. McClintock, R. Shafee, R. Narayan, R. A. Remillard, S. W. Davis, and L.-X. Li, Astrophys. J. 652, 518 (2006).

[8] K. A. Postnov and L. R. Yungelson, Living Rev. Relativity 9, 6 (2006).

[9] V. Kalogera, Pramana 63, 673 (2004).

[10] J. Abadie et al. (LIGO Scientific Collaboration and Virgo Collaboration), Classical Quantum Gravity 27, 173001 (2010).

[11] T. A. Apostolatos, C. Cutler, G. J. Sussman, and K. S. Thorne, Phys. Rev. D 49, 6274 (1994).

[12] J. Aasi et al. (LIGO Scientific Collaboration and Virgo Collaboration), Phys. Rev. D 88, 062001 (2013).

[13] J. Abadie et al. (LIGO Scientific Collaboration and Virgo Collaboration), Phys. Rev. D 83, 122005 (2011); J. Abadie et al. (LIGO Scientific Collaboration and Virgo Collaboration), ibid. 86, 069903(E) (2012).

[14] J. Aasi et al. (LIGO Scientific Collaboration and Virgo Collaboration), Phys. Rev. D 87, 022002 (2013).

[15] J. Abadie et al. (LIGO Scientific Collaboration and Virgo Collaboration), Phys. Rev. D 85, 082002 (2012).

[16] B. Allen, Phys. Rev. D 71, 062001 (2005).

[17] B. Abbott et al. (LIGO Scientific Collaboration), Phys. Rev. D 78, 042002 (2008).

[18] C. Van Den Broeck, D. Brown, T. Cokelaer, I. Harry, G. Jones, B. Sathyaprakash, H. Tagoshi, and H. Takahashi, Phys. Rev. D 80, 024009 (2009).

[19] I. W. Harry and S. Fairhurst, Classical Quantum Gravity 28, 134008 (2011).

[20] D. Fazi, Ph.D. thesis, Universita di Bologna, 2009.

[21] A. Buonanno, Y. Chen, and M. Vallisneri, Phys. Rev. D 67, 104025 (2003); A. Buonanno, Y. Chen, and M. Vallisneri, ibid. 74, 029904(E) (2006).

[22] Y. Pan, A. Buonanno, Y.-b. Chen, and M. Vallisneri, Phys. Rev. D 69, 104017 (2004); Y. Pan, A. Buonanno, Y.-b. Chen, and M. Vallisneri, ibid. 74, 029905(E) (2006).

[23] D. Brown, A. Lundgren, and R. O’Shaughnessy, Phys. Rev. D 86, 064020 (2012).
[24] D. Brown, I. Harry, A. Lundgren, and A. Nitz, Phys. Rev. D 86, 084017 (2012).

[25] I. Harry, A. Nitz, D. Brown, A. Lundgren, E. Ochsner, and D. Keppel, arXiv:1307.3562.

[26] P. Ajith, N. Fotopoulos, S. Privitera, A. Neunzert, and A. J. Weinstein, arXiv:1210.6666.

[27] B. Abbott et al. (LIGO Scientific Collaboration), Phys. Rev. D 77, 062002 (2008).

[28] C. A. K. Robinson, B. S. Sathyaprakash, and A. S. Sengupta, Phys. Rev. D 78, 062002 (2008).

[29] T. Cokelaer, Phys. Rev. D 76, 102004 (2007).

[30] B. J. Owen, Phys. Rev. D 53, 6749 (1996).

[31] B. J. Owen and B. S. Sathyaprakash, Phys. Rev. D 60, 022002 (1999).

[32] B. Sathyaprakash, Phys. Rev. D 50, R7111 (1994).

[33] I. W. Harry, B. Allen, and B. S. Sathyaprakash, Phys. Rev. D 80, 104014 (2009).

[34] G. M. Manca and M. Vallisneri, Phys. Rev. D 81, 024004 (2010).

[35] P. Ajith et al., Phys. Rev. Lett. 106, 241101 (2011).

[36] LSC Algorithm Library software packages LAL, LALWRAPPER, and LALAPPS, http://www.lsc-group.phys.uwm.edu/lal.

[37] K. Cannon et al., Astrophys. J. 748, 136 (2012).

[38] G. M. Harry (LIGO Scientific Collaboration), Classical Quantum Gravity 27, 084006 (2010).

[39] Advanced Virgo Baseline Design (2009), VIR027A09, https://pub3.ego-gw.it/itf/tds/file.php?callFile=VIR-0027A09.pdf.

[40] T. A. Apostolatos, Phys. Rev. D 52, 605 (1995).

[41] B. Allen, W. G. Anderson, P. R. Brady, D. A. Brown, and J.D.E. Creighton, Phys. Rev. D 85, 122006 (2012).

[42] B. Abbott et al. (LIGO Scientific Collaboration), Phys. Rev. D 79, 122001 (2009).

[43] J. Abadie et al. (LIGO Scientific Collaboration), Phys. Rev. D 83, 122005 (2011).

[44] A. Buonanno, B. R. Iyer, E. Ochsner, Y. Pan, and B. S. Sathyaprakash, Phys. Rev. D 80, 084043 (2009).

[45] A. Lazzarini and R. Weiss, Report No. LIGO-E950018-02-E, 1996.

[46] J. Abadie et al. (LIGO Scientific Collaboration and Virgo Collaboration), arXiv:1003.2481.

[47] P. Ajith, Phys. Rev. D 84, 084037 (2011).

[48] C. Hanna, Ph.D. thesis, Louisiana State University, 2008.

[49] J. Aasi et al. (LIGO Collaboration and Virgo Collaboration), Phys. Rev. D 88, 062001 (2013).

[50] M. Campanelli, C. Lousto, and Y. Zlochower, Phys. Rev. D 74, 041501 (2006). 